\title{
Infantile Case of Pneumomediastinum Complicated by Epidural Pneumatosis
}

\author{
Yohei Sato $^{\mathrm{a}, \mathrm{b}, \mathrm{c}}$, Chikara Otsubo ${ }^{\mathrm{a}}$, Yasuyuki Wada ${ }^{\mathrm{a}}$, Masakatsu Kubo ${ }^{\mathrm{a}}$, Hiroyuki Ida ${ }^{\mathrm{b}}$
}

\begin{abstract}
Epidural pneumatosis is a particularly rare condition in which there is air in the epidural space. We report a 1-year-old infant with spontaneous pneumomediastinum complicated by epidural pneumatosis, which is one of the most severe complications. Even though the patient had an upper respiratory infection, no other preceding factor was found. He did not show any neurological symptoms and he improved with conservative treatment. Early diagnosis of epidural pneumatosis is important to avoid further neurological complications.
\end{abstract}

Keywords: Epidural pneumatosis; Pneumorrhachis; Pneumomediastinum; Subcutaneous emphysema; Child

\section{Introduction}

Epidural pneumatosis is a particularly rare condition in which there is air in the epidural space and it occasionally causes neurological complications such as hemiplegia [1]. It usually occurs as a severe complication of pneumomediastinum and subcutaneous emphysema [2]. Several cases of epidural pneumatosis were reported to be complicated by asthma attacks, diabetic ketoacidosis or trauma [3-5]. We report here a case of epidural pneumatosis in a previously healthy infant without any reported risks and its spontaneous resolution.

Manuscript accepted for publication March 31, 2014

\footnotetext{
${ }^{a}$ Department of Pediatrics, Jikei University Kashiwa Hospital, Chiba, Japan

${ }^{\mathrm{b}}$ Department of Pediatrics, Jikei University School of Medicine, Tokyo, Japan

${ }^{\mathrm{c} C}$ Corresponding author: Yohei Sato, Department of Pediatrics, Jikei University School of Medicine, 3-25-8 Nishishimbashi Minato-ku, Tokyo 105-8461, Japan. Email: yoheisato@jikei.ac.jp
}

doi: http://dx.doi.org/10.14740/ijcp146w

\section{Case Report}

A 1-year-old previous healthy infant was admitted to the hospital for sudden dyspnea. He started to cough 2 days before admission, although he had been doing well for 2 days. He suddenly developed dyspnea and lethargy at home. His mother denied any past medical history of asthma, diabetes mellitus or foreign body ingestion.

The patient's body temperature was $37.9^{\circ} \mathrm{C}$, pulse rate was 159 beats/min, respiratory rate was 44 breaths/min and blood pressure was $120 / 88 \mathrm{mmHg}$. Oxygen saturation was $95 \%$ at room air and $100 \%$ on oxygen. On physical examination, there were decreased breathing sounds on the left side and crepitation on the chest wall. He was alert and did not show any abnormal neurological findings.

Laboratory data showed an elevated white blood cell count of $13850 / \mu \mathrm{L}$, and a differential showed $56.5 \%$ polymorphonuclear leukocytes. The C-reactive protein $(3.09 \mathrm{mg} /$ $\mathrm{dL}$ ) level was elevated. Other laboratory values were within normal limits. Bacterial culture results of blood, nose and throat were negative.

A chest radiograph demonstrated hyper translucency in the left upper lobe (Fig. 1). Computed tomography (CT) was

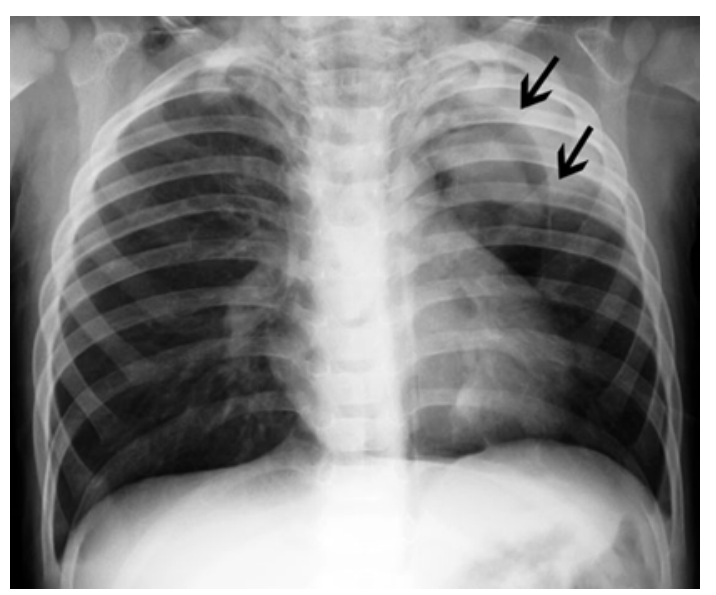

Figure 1. Chest radiograph shows pneumomediastinum with subcutaneous emphysema. 

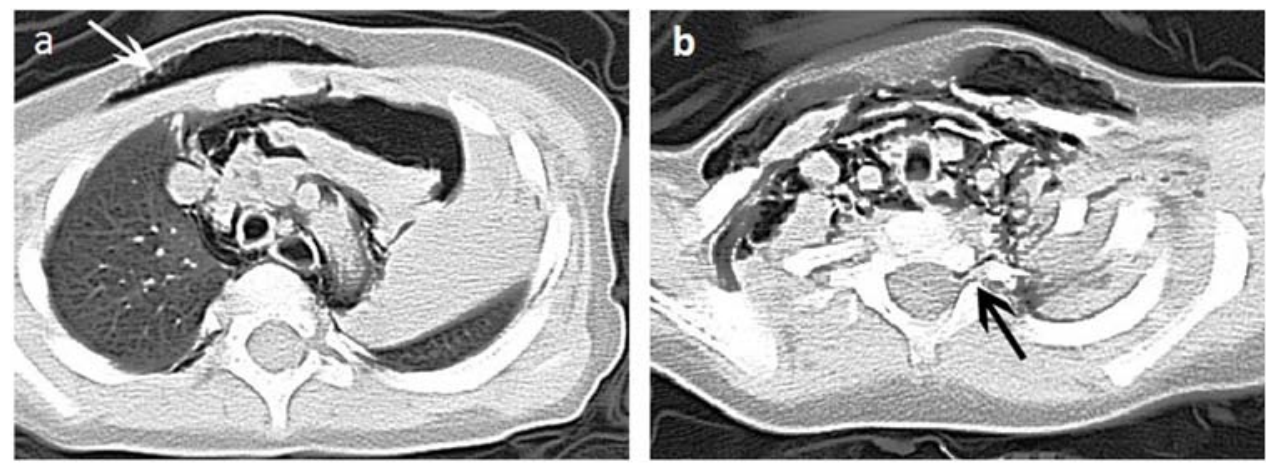

Figure 2. Thoracic axial CT images show (a) pneumomediastinum and subcutaneous emphysema (b) epidural pneumatosis.

performed for further investigation and it showed massive pneumomediastinum and subcutaneous emphysema (Fig. 2a). At the same time, there was the air entering through the cervical spine into the epidural space (Fig. 2b).

The patient was hospitalized for close monitoring and treatment. We allowed him to rest and gave high flow oxygen therapy. Intravenous antibiotics (ABPC/SBT) were administered. His condition rapidly improved. A CT scan was repeatedly taken 2 days after admission and air in the epidural space was observed. Pneumomediastinum and subcutaneous emphysema were remarkably improved within 1 week. The patient did not show any neurological symptoms during hospitalization. He was discharged on the 12th hospital day without any other complications. In the follow-up, he has been doing well and there has not been any recurrence.

\section{Discussion}

Spontaneous pneumomediastinum is defined as the condition in which there is air in the mediastinum without any history of trauma, cardiac surgery or mechanical ventilation [1]. It usually occurs among older children and adolescents; however, some infantile cases have been reported to be associated with respiratory infection [6]. In our case, pneumomediastinum suddenly developed during the course of upper respiratory infection. Spontaneous pneumomediastinum has been considered to be benign because most cases resolve by themselves; however, some severe complications have been reported [7].

Epidural pneumatosis is a particularly rare condition in which there is the air in epidural space and it is usually secondary to pneumomediastinum. Although epidural pneumatosis might be complicated by asthma attacks, diabetic ketoacidosis or trauma [3-5],there are no preceding events other than pneumomediastinum, and it might have spontaneously occurred in our patient.

Even though epidural pneumatosis is one of the most severe complications of pneumomediastinum, it is also con- sidered as a benign condition because it is usually self-limiting and does not require any surgical procedure. Because neurological symptoms might occur if epidural pneumatosis left untreated, it should be excluded in massive pneumomediastinum. A chest radiograph is the standard diagnostic modality for pneumomediastinum, but it is not always able to detect epidural pneumatosis. Therefore, a CT scan is also performed as an appropriate alternative.

In our patient, the progression to epidural pneumatosis was so rapid that we could not determine the exact timing at which pneumomediastinum progressed to epidural pneumatosis. Our patient did not manifest neurological symptoms, but it is important to monitor neurological deterioration during pneumomediastinum.

In conclusion, we report infantile epidural pneumatosis secondary to spontaneous pneumomediastinum. We did not find any previously reported risk factors in our case. Epidural pneumatosis and pneumomediastinum improved with watchful waiting. A CT scan is effective for diagnosis and it should be considered in massive pneumomediastinum. Neurological deterioration should be monitored during the clinical course of pneumomediastinum. Early diagnosis is important to avoid neurological complications and further morbidity in epidural pneumatosis.

\section{Conflict of Interest}

None.

\section{References}

1. Chalumeau M, Le Clainche L, Sayeg N, Sannier N, Michel JL, Marianowski R, Jouvet P, et al. Spontaneous pneumomediastinum in children. Pediatr Pulmonol. 2001;31(1):67-75.

2. Dontu VS, Kramer D. Spontaneous pneumothorax, pneumomediastinum, and epidural emphysema pre- 
senting as neck pain suspicious for meningitis. Pediatr Emerg Care. 2007;23(7):469-471.

3. Ersoy B, Polat M, Coskun S. Diabetic ketoacidosis presenting with pneumomediastinum. Pediatr Emerg Care. 2007;23(1):67.

4. Gibikote S, Wray A, Fink AM. Pneumorrhachis secondary to traumatic pneumomediastinum in a child. Pediatr Radiol. 2006;36(7):711-713.

5. Harrod CC, Boykin RE, Kim YJ. Epidural pneumato- sis of the cervicothoracic spine associated with transient upper motor neuron findings complicating Haemophilus influenzae pharyngitis, bronchitis, and mediastinitis. J Pediatr Orthop. 2010;30(5):455-459.

6. McNicholl B. Pneumomediastinum and subcutaneous emphysema in status asthmaticus, requiring surgical decompression. Arch Dis Child. 1960;35:389-392.

7. Stack AM, Caputo GL. Pneumomediastinum in childhood asthma. Pediatr Emerg Care. 1996;12(2):98-101. 